\title{
The use of braces in the rehabilitation treatment of the post-traumatic elbow
}

\author{
ISABELLA FUSARO ${ }^{1}$, STEFANIA ORSINI ${ }^{1}$, TERESA SFORZA ${ }^{1}$, ROBERTO ROTINI', \\ MARIA GRAZIA BENEDETTI ${ }^{1}$
}

${ }^{1}$ Physical Medicine and Rehabilitation Unit, Rizzoli Orthopaedic Institute, Bologna, Italy

${ }^{2}$ Shoulder and Elbow Surgery Unit, Rizzoli Orthopaedic Institute, Bologna, Italy

\begin{abstract}
The elbow, the middle joint of the upper limb, enables the hand to be placed at various distances from the body. The articular, muscular and neuromotor complexity of the elbow is such that it is often prone to stiffness, especially if immobilized for long periods of time. Therefore, mobilization of the posttraumatic elbow must be started early. In the presence of lesions to the ligamentous structures, the joint must be protected to prevent these structures from being placed under tension during movement. Rehabilitation of the elbow includes the use of braces. Different types of brace are used depending on clinical situation and the objectives to be achieved. They can have fixed protection or locked articulation, allow an adjustable range of movement to restrict flexion-extension and pronation-supination, or allow dynamic and static progressive movement. The latter plays a special role in conservative treatment and following surgical release. However, the effectiveness of braces in the rehabilitation treatment of elbow stiffness depends on the patient's compliance. Their use requires a considerable amount of physician time to achieve the objectives envisaged by the rehabilitation program.
\end{abstract}

Key Words: brace, elbow, rehabilitation, stiffness, trauma.

\author{
Corresponding Author: \\ Maria Grazia Benedetti, MD \\ Physical Medicine and Rehabilitation Unit, Rizzoli \\ Orthopaedic Institute \\ Via G. C. Pupilli 1, 40136 Bologna, Italy \\ Phone: +390516366236 \\ Fax: +39051332392 \\ E-mail: benedetti@ior.it
}

\section{Introduction}

The elbow, the middle joint of the upper limb, enables the hand to be placed at varying distances from the body (1). In the elbow, peri-articular muscles and soft tissues interact in a complex way to maintain joint homeostasis and regulate movement through proprioceptive feedback. Flexion-extension movements regulate the length of the limb, allowing the hand to be brought to the mouth or moved in the space around the body. Pronation-supination movements (rotating the palm of the hand) facilitate the reaching and grasping of objects. Due to its articular, muscular and neuromotor complexity, the elbow is often prone to stiffness, especially if, following an injury or operation, it is immobilized for a long period of time (2). Indeed, immobilization alters the orientation and mechanical properties of the peri-articular soft tissues (3), actin-myosin binding and peripheral afferent nerve stimulation. Therefore, in elbow rehabilitation, mobilization must be started early; in the presence of combined lesions of the ligamentous structures, the joint must be protected to prevent these structures from being placed under tension during movement. Furthermore, the rehabilitation treatment must respect the phases of histological healing and, accordingly, have different objectives in each phase. In particular, in the inflammatory phase the aim is to control pain and swelling, in the fibroblastic phase it is to recover range of movement (ROM) and muscle flexibility, while in the remodeling phase the aim is to recover neuromuscular control (4).

The rehabilitation program includes the use of elbow braces. These are used with different aims according to the pathology, the phase of treatment and the final objective of the treatment. 


\section{Classification of elbow braces}

Since elbow braces are used for different clinical situations and objectives, there exist several different types. According to Jacobs (5), braces can have fixed protection or locked articulation, allow an adjustable ROM to restrict flexion-extension and pronation-supination, or allow dynamic and static progressive movement (Tab. 1). Furthermore, braces can be customized or

Table 1. Classification of elbow braces (5).

\begin{tabular}{ll}
\hline Protective braces & Fixed \\
& With locked articulation \\
Braces with adjustable ROM & Limitation of flexion/extension \\
& Limitation of pronation/supination \\
Mobilization braces & $\begin{array}{l}\text { Dynamic } \\
\text { Static/progressive }\end{array}$ \\
\hline
\end{tabular}

ready-made. In the past the most commonly used materials for customized braces were metal alloys. Currently, thermoplastic materials (polyethylene or polypropylene) are the ones mainly used, in thicknesses ranging from 2 to $5 \mathrm{~mm}$ according to the required stiffness and strength. Braces manufactured with this type of material can be combined with straps or with unhinged, locked or progressively adjustable mechanical joints. Splint tuners are mostly made of metal and serve to lock elbow flexion-extension at a set level. The simplest ready-made braces are splints made of cardboard or cork which are fixed in place with elastic bands. Cork braces, because of the flexibility of this material, allow small movements of the elbow and are therefore preferable in order to avoid excessive immobilization. Ready-made braces mostly consist of an aluminum frame, which can be combined with a joint, padding and straps. The joint may be the unrestrained or "ROM-type", i.e. with a knob or Allen key for manual adjustment of the ROM. The most commonly used paddings are silicone or neoprene; they help to avoid sores which would occur with direct contact between the limb and metal frame.

The straps generally used are the kind with Velcro fasteners or press-studs. The straps serve to secure the brace to the limb and prevent it slipping out of place. In some cases a shoulder strap is provided to support the limb.

\section{Protective braces}

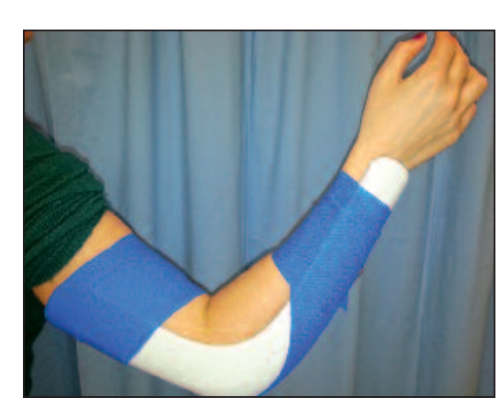

Fig. 1. Protective brace in unhinged flexion.
Protective braces, unhinged and hinged with locked articulation, protect the elbow by preventing its movement altogether in the immediate post-injury or postoperative periods. They are used to reduce edema and pain in the inflammatory phase and to promote repair of the damaged capsule-ligament structures and bone. In the protective brace the elbow may be immobilized in flexion (Fig. 1) or extension and the forearm in pronation or supination or intermediate positions. Protective braces are indicated in the initial phases of fracture-dislocation treatment or in the immediate post-operative period.

\section{Braces with adjustable ROM}

Braces with adjustable ROM (Fig. 2) allow protected flexion-extension movement. They are used after an injury or in the post-operative period to allow early movement without damaging the healing tissue. In particular, in elbow fractures, treatment with braces should take into account the possible presence of a collateral ligament lesion and the histological healing phase (6).

If the ligaments are intact, an elbow brace is set at $90^{\circ}$ of flexion in the acute phase, with the forearm in intermediate rotation. After 1-7 days, unrestrained mobilization is gradually started in flexion-extension and pronation-supination and the elbow is put back in the brace at the end of the exercises. After 7-15 days, the brace is removed and mobilization exercises are performed up to recovery of complete ROM.

In cases of Ulnar Lateral Collateral Ligament (ULCL) injury, the elbow, in the acute phase, should be placed in a brace at $90^{\circ}$ of flexion with the forearm in pronation. After 1-7 days, protected mobilization can be started in flexion-extension with the forearm in pronation and mobilization in pronation-supination with 


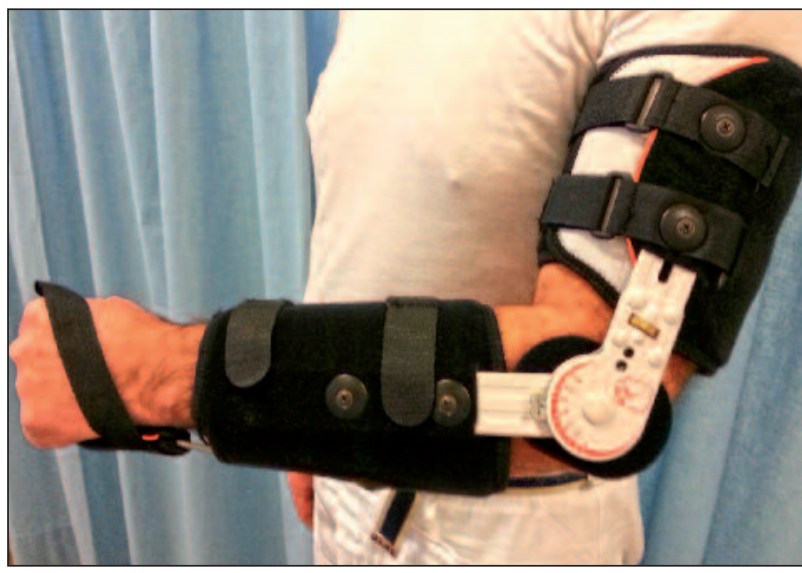

Fig. 2. Adjustable range of motion brace (forearm in any position).

the elbow flexed. For six weeks, full extension in supination and abduction of the shoulder combined with intra-rotation should be avoided without a brace. In cases of Medial Collateral Ligament (MCL) injury, the elbow should be placed in a brace at $90^{\circ}$ of flexion with the forearm in supination. After 1-7 days, protected mobilization in flexion-extension with the forearm supine and mobilization in pronation-supination with the elbow flexed can be started. For six weeks, full extension in pronation should be avoided.

Finally, in cases of injury to both the ULCL and the MCL, the elbow should be placed in a brace flexed at $90^{\circ}$ with the forearm in an intermediate position. After 1-7 days, protected mobilization in flexionextension in intermediate rotation and mobilization in pronation-supination with the elbow flexed can be started. For six weeks, maximum extension in supination or pronation should be avoided.

\section{Mobilization braces in flexion-extension: dynamic and static}

Mobilization braces exploit the viscoelastic properties of soft tissues by producing a slow, gradual and progressive distraction alternated with a phase of rest in tension. These small increases in ROM held for a long time enable the length of the soft tissues to be altered. Dynamic progressive mobilization braces (Fig. 3) produce a continuous stimulation that, over time, might lead to an inflammatory process. Thus, static progressive braces, exerting stimulation alternated with rest,
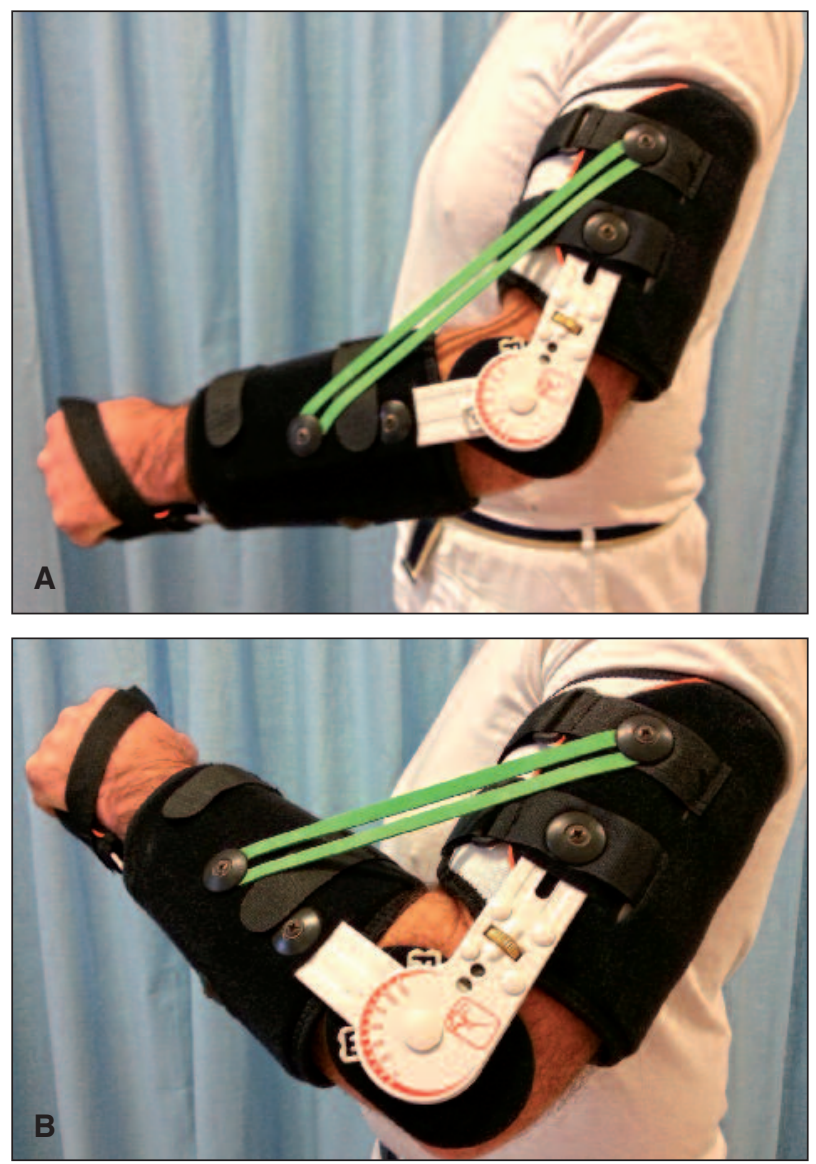

Fig. 3. Dynamic progressive brace in flexion: initial position (A) and final results $(B)$.

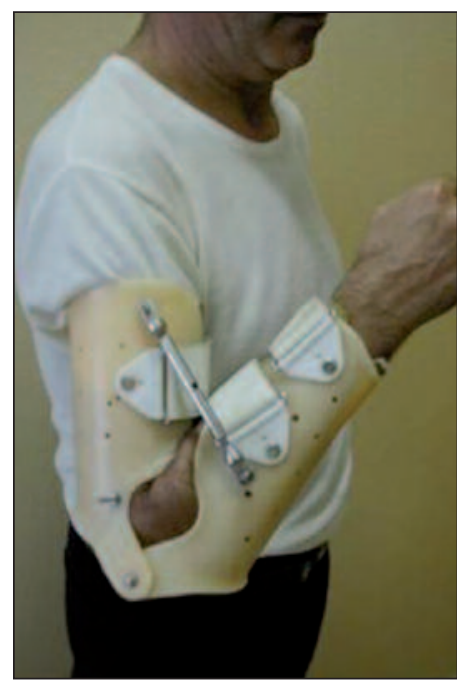

Fig. 4. Static progressive mobilization brace with splint tuner. are preferred as they allow biological adaptation of the tissue. Static progressive braces include those equipped with tuners (Fig. 4) that produce distraction forces on the humeroulnar joi$n t$ in the final degrees of extension and compression forces in the final degrees of flexion. Conversely, mobilization braces with a knob or key (Fig. 5) have a system to control the articu- 


\section{oints}

I. Fusaro et al.

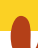

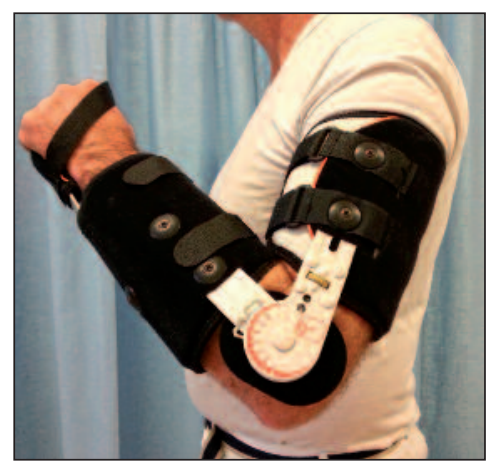

Fig. 5. Static progressive mobilization brace with key and tension in flexion.

when the articulation is completely locked, a brace with an adjustable ROM when the joint is set to allow a limited range of movement and a mobilization brace when the articulation is locked in tension.

\section{Indications for the use of mobilization braces in flexion-extension}

Mobilization braces, together with rehabilitation, play an important role in the conservative treatment of the stiff elbow and in post-surgical treatment after arthroscopic release.

Elbow stiffness depends on articular congruence, capsuloligamentous structures, peri-articular muscles (in particular the brachialis anterior) and pain which entails a voluntary and inadvertent reduction in ROM. Pain appears in $25 \%$ of high-energy traumas, $15 \%$ of dislocations and also in low-energy traumas with immobilization and it is the most common complication after a trauma involving fracture, dislocation or damage to soft tissues $(7,8)$. Elbow stiffness is a very disabling complication because it impedes the fundamental role of the elbow: placing the hand in space. Furthermore, elbow stiffness is rarely accepted by the patient due to the lack of useful compensation in the over- and underlying joints (9). A 50\% reduction of elbow ROM can cause an at least $80 \%$ loss of function of the upper limb (10). In agreement with Morrey (11), a flexion-extension of $30^{\circ}-130^{\circ}$ and a pronation-supination of $50^{\circ}-50^{\circ}$ constitute the necessary functional ROM of the elbow for activities of daily living (ADL).

Elbow stiffness is difficult to treat; in order to choose the most suitable treatment we need to know if the stiffness is extrinsic, i.e. extra-articular, due to capsular contraction, ossifications and osteophytes, or intrinsic, i.e. intra-articular, due to malunion, cartilage defect, arthritis, or mixed causes. The conservative treatment of stiffness with a brace and rehabilitation requires considerable determination on the part of the patient and is indicated for moderate extrinsic stiffness, i.e. with a range of movement of between $60^{\circ}$ and $90^{\circ}$, with elastic arrest, provided that the stiffness has not be present for longer than 6-12 months. Conversely, conservative treatment is contraindicated in the presence of very severe intrinsic stiffness (ROM less than $30^{\circ}$ ), severe stiffness (ROM between $30^{\circ}-60^{\circ}$ ), heterotopic ossifications, and neuropathy of the ulnar nerve and also if the patient is not highly motivated. The brace is used to correct tension because insufficient tension produces ineffective lengthening, whereas excessive tension can produce contractures, hemorrhage, adhesions, ossifications and further stiffness. Furthermore, the brace is used to avoid a feeling of painful tension and must not cause numbness, skin color changes or a feeling of intolerance. According to the protocol of conservative treatment for elbow stiffness proposed by Morrey (11), the brace, allowing only the most necessary movement, is worn from midnight until 8 a.m.; during the day the position is changed every 4 hours, integrating active movement each time the brace is removed. The brace is worn for 3-4 months up to one year and thereafter gradually suspended.

A recent review (12) analyzed 13 studies, which included a total of 247 patients with post-traumatic elbow stiffness, treated with static, static progressive and dynamic braces for a mean of $6.9 \pm 5.1$ months. It was found that each type of brace analyzed was effective in achieving an improvement in ROM, with a mean increase of $38^{\circ} \pm 10^{\circ}$. The protocols that involved the use of dynamic braces achieved the greatest increase in ROM $\left(46^{\circ} \pm 10^{\circ}\right)$, followed by those that involved the use of static progressive braces $\left(40^{\circ} \pm 10^{\circ}\right)$ and static braces $\left(34^{\circ} \pm 10^{\circ}\right)$. The authors concluded that conservative treatment of post-traumatic elbow stiffness with braces is an effective option, which also ensures results similar to those obtained by surgical techniques, yet without the complications connected with surgery. Although there emerged no statistically significant dif- 
ferences between the outcomes of the three protocols, the static progressive brace is the first choice because it seems to improve patient compliance (12).

As regards the treatment of stiffness after surgical release, the greatest difficulty is not the surgical resolution of adhesions and retraction, but how to maintain the surgically restored ROM; there is ample literature on the surgical techniques available, but the literature on post-operative regimens is scarce. The treatment of stiffness with a brace after surgical release is facilitated by locoregional analgesics and it involves wearing the mobilization brace during the day. The brace is worn in maximum flexion or extension for 40 minutes of every hour, and continuous passive motion exercises are performed during the other 20 minutes with the brace off (13).

\section{Indications for the use of mobilization braces in pronation-supination}

Mobilization braces in pronation-supination have been described less in the literature because the recovery of elbow movement in this plane is easier to achieve. However, when there is a deficit of pronationsupination that restricts functional activities or when more pronation-supination ROM than functional ROM is required for work or sports purposes, mobilization braces can be used in pronation-supination. The commercially available ready-made braces include a brace consisting of an aluminum modular structure. Two plastic grips are applied to the aluminum structure, one for the arm and one for the forearm-wristhand, both adjustable by velcro straps. Furthermore, at the wrist and elbow level there are two knobs for adjusting the flexion-extension of elbow and the pronation-supination of the forearm-wrist-hand, respectively. Although this brace offers the advantage of allowing gradual recovery of pronation-supination, it has several disadvantages; for example, it is bulky, difficult to put on, impractical for carrying out ADL, and unsuitable for use at night.

Another ready-made brace for the recovery of pronation-supination consists of a rigid aluminum structure with posterior rings, padding and straps for covering and fixing (Fig. 6). Both flexion-extension of the
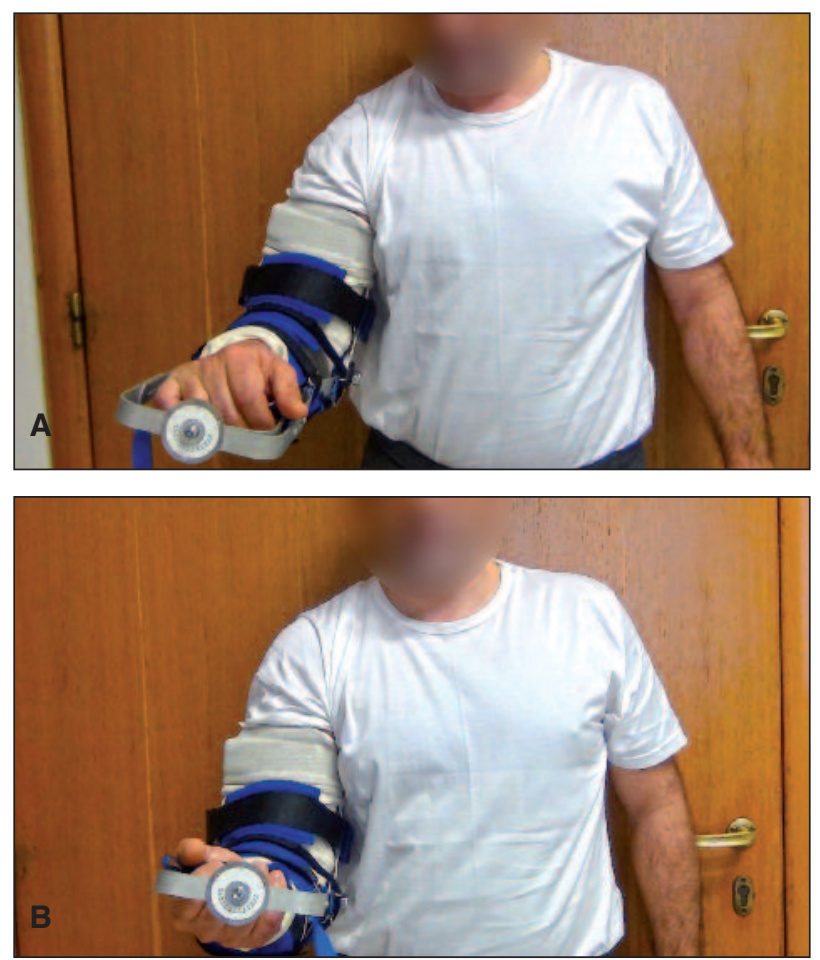

Fig. 6. Mobilization brace in pronation-supination with aluminum bars in pronation $(A)$ and in supination $(B)$.

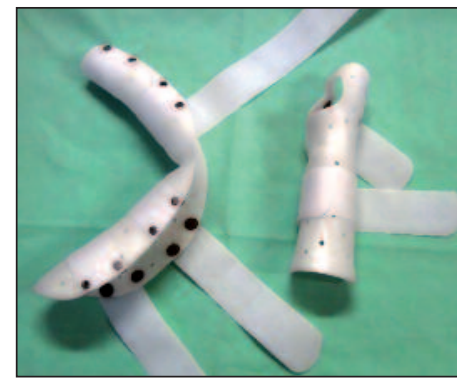

Figure 7. Mobilization brace in pronation-supination customized with overlapping position splints. elbow and pronationsupination can be adjusted by means of a special key on a handle. With respect to the previously described brace this one has the advantage of being less bulky and easier to use in the daytime. However, this brace has sev- eral disadvantages, including the poor quality of the materials used in manufacturing, the difficulty in putting it on, and the poor adhesion of the brace when it is placed in tension. As a result, the set objectives are difficult to achieve and the patient's compliance is reduced.

On the basis of these considerations the authors have developed a customized mobilization brace for restoring pronation-supination. It is made up of two splints, unhinged, which overlap, one sliding over the other (Fig. 7). The arm-forearm splint is the first to be 

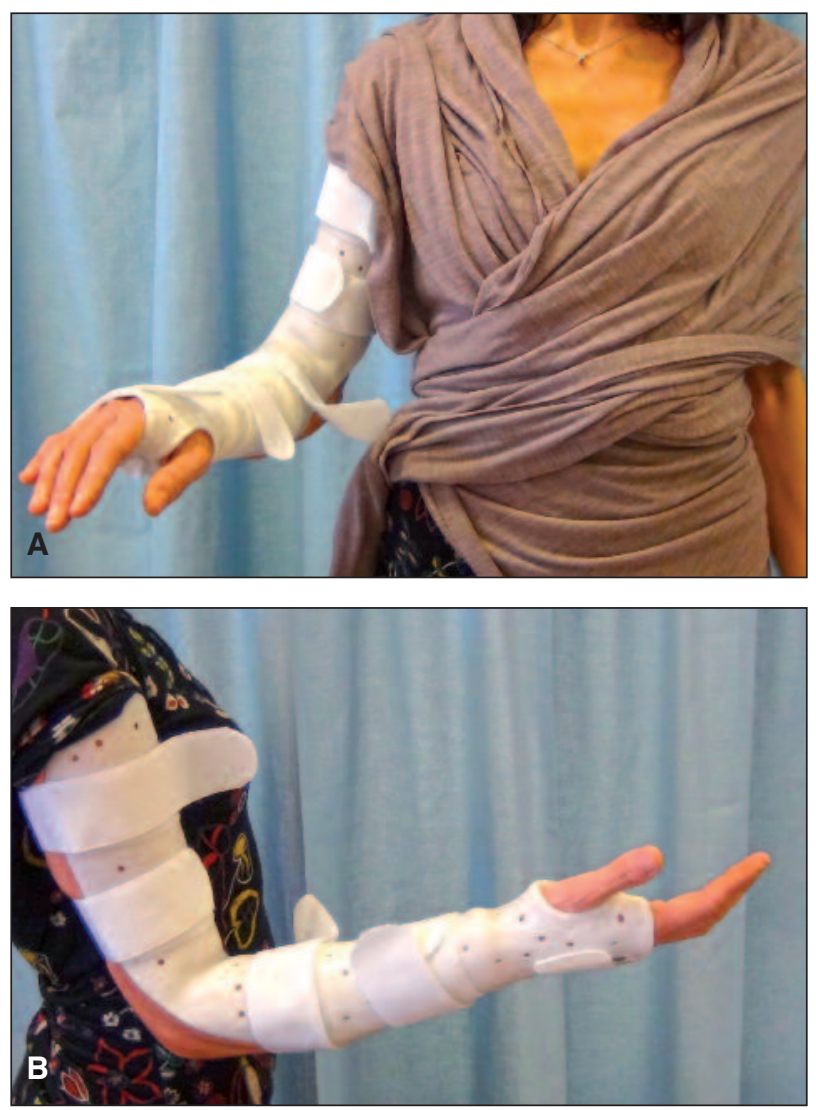

Figure 8. Customized mobilization brace in pronation $(A)$ and in supination (B).

placed and it holds the elbow at $90^{\circ}$ of flexion, then the forearm-wrist-hand splint is placed, leaving the inter-phalangeal joints free to move (Fig. 8).

Pronation-supination is corrected by rotating the second splint on the first and the position reached is fixed using velcro straps. There are several advantages to this type of brace with respect to the previously described models. First, patient compliance is greater using this brace because it is easier to wear, light, and can be used at the night with less risk of positional hyperpressure. Furthermore, being customized it is unique to the patient, unlike previous ready-made models. This brace is worn full time, switching between pronation and supination when both these movements are insufficient; instead it is worn full time only in pronation or supination when just one of these two movements is insufficient. The patient is instructed to remove the brace several times a day to perform elbow movement exercises in all planes.

\section{Conclusions}

Braces are useful in elbow rehabilitation after acute injury or surgical fixation to decrease the risk of venous stasis and complex regional pain syndrome and to allow movement within a safe ROM. In the presence of stiffness they can constitute the basis of conservative treatment. After surgical release, they are used to maintain the ROM obtained in the operating room. The disadvantages of applying mobilization braces are their cost, the physician time necessary for explaining to patients how to use them and for assessing the results obtained, and problems of patient compliance.

\section{References}

1. Jupiter JB, O'Driscoll SW, Cohen MS. The assessment and management of the stiff elbow. Instr Course Lect. 2003;52: 93-111.

2. Bruno RJ, Lee ML, Strauch RJ, et al. Posttraumatic elbow stiffness: evaluation and management. J Am Acad Orthop Surg. 2002;10:106-116.

3. Cohen MS, Schimmel DR, Masuda K, et al. Structural and biochemical evaluation of the elbow capsule after trauma. J Shoulder Elbow Surg. 2007;16:484-490.

4. Wilk KE, Arrigo C, Andrews JR. Rehabilitation of the elbow in the throwing athlete. J Orthop Sports Phys Ther. 1993; 17:305-317.

5. Jacobs ML. Splint classification. In: Jacobs ML, Austin GP (Eds), Splinting the Hand and Upper Extremity. Philadelphia, Lippincott Williams and Wilkins. 2003;2-18.

6. O'Driscoll SW, Jupiter JB, King GJ, et al. The unstable elbow. Instr Course Lect. 2001;50:89-102.

7. Chinchalkar SJ, Szekeres M. Rehabilitation of elbow trauma. Hand Clin. 2004;20:363-374.

8. MacDermid JC, Vincent JI, Kieffer L, et al. A survey of practice patterns for rehabilitation post elbow fracture. Open Orthop J. 2012;6:429-439.

9. Marinelli A, Bettelli G, Guerra E, et al. Mobilization brace in post-traumatic elbow stiffness. Musculoskelet Surg. 2010;94 (Suppl 1): S37-S45.

10. Søjbjerg JO. The stiff elbow. Acta Orthop Scand. 1996; 67:626-631.

11. Morrey BF. Splints and bracing at the elbow. In: Morrey BF (Ed.). The Elbow and its Disorders, 3rd edn. Philadelphia, Saunders. 2000;50-154.

12. Müller AM, Sadoghi P, Lucas R, et al. Effectiveness of bracing in the treatment of nonosseous restriction of elbow mobility: a systematic review and meta-analysis of 13 studies. J Shoulder Elbow Surg. 2013;22:1146-1152.

13. O'Driscoll SW, Giori NJ. Continuous passive motion (CPM). Theory and principles of clinical application. J Rehabil Res Dev. 2000;37:179-188. 